\title{
Valores presentes en el paisaje de la ciudad de Pachuca, Hidalgo, México
}

\author{
Values present in the landscape of the city \\ of Pachuca, Hidalgo, Mexico
}

María Elena Sánchez-Roldán* ๑ https://orcid.org/0000-0003-1802-8370

Citar este artículo como: Sánchez-Roldán, M.E. (2020). Valores presentes en el paisaje de la ciudad de Pachuca, Hidalgo, México. Revista Nodo, 15 (29), pp. 87-98.

\section{Resumen}

Esta investigación forma parte del trabajo de tesis realizado para obtener el grado de doctora en Diseño, Planificación y Conservación de Paisajes y Jardines, en la Universidad Autónoma Metropolitana, Unidad Azcapotzalco, cuyo título es Revaloración del Patrimonio Cultural de la Ciudad de Pachuca, Hidalgo, México. Antes de abordar el concepto de revaloración, es necesario definir qué es un valor y cómo éste es identificado dentro de los componentes del paisaje de la ciudad; por ello se pretende describir los valores del paisaje presentes en la zona en estudio, que hacen de esta metrópoli un sitio icónico por su historia, riqueza mineral, topografía y cultura. Se ha tomado como referente el método de análisis de la Metropolitan Situation Analysis (MESA), concebido por el programa "Training for Education, Learning and Leadership Towards a New Metropolitan discipline TELLme", perteneciente a la red internacional de investigación sobre ciudades y metrópolis, coordinada por la Facultad de Arquitectura del Politécnico de Milán, Italia, a través del Programa Erasmus Plus de la Unión Europea, 2014-2020 y el documento Catálogo de Paisaje de la Región Metropolitana de
Barcelona (2017) así como una exhaustiva recopilación de información documental, bibliográfica, cartográfica y fotográfica y realización de recorridos por la ciudad, todo ello con miras a recabar información que permita la apreciación del Patrimonio Cultural de la ciudad a través de los valores paisajísticos presentes en ella.

Palabras clave: Valores, Paisaje, Ciudad, Patrimonio cultural.

\section{Abstract}

This research is part of the thesis work done to obtain a PhD in Design, Planning and Conservation of Landscapes and Gardens, at the Autonomous Metropolitan University, Azcapotzalco Unit, whose title is: Revaluation of the Cultural Heritage of the City of Pachuca, Hidalgo, Mexico. However, before being able to approach the concept of revaluation, it is necessary to define what a value is and how it is identified within the components of the city landscape. So, it is intended to describe the values of the landscape present in the study area, which make this metropolis an iconic site for its history, mineral wealth, topography, and culture.

Fecha de recepción: 12 de mayo de 2020 • Fecha de aceptación: 25 de julio de 2020

\footnotetext{
* Arquitecta. Candidata a doctora en Diseño, Planificación y Conservación de Paisajes y Jardines en la Universidad Autónoma Metropolitana, Unidad Azcapotzalco, México y profesora investigadora en la Universidad Autónoma del Estado de Hidalgo. Correo electrónico: mariaesr@uaeh.edu.mx
} 
The method of the Metropolitan Situation Analysis (MESA), conceived by the program "Training for Education, Learning and Leadership Towards a New Metropolitan discipline Tell me", belonging to the international network of research on cities and metropolises, has been taken as a reference. coordinated by the Faculty of Architecture of the Polytechnic of Milan, Italy, through the Erasmus Plus Program of the European Union, 2014-2020 and the document of the Catalog of Landscapes of the Barcelona Metropolitan Region (2017) as well as an exhaustive compilation of documentary, bibliographic, cartographic and photographic information, as well as tours of the city, all with a view to disseminating information that allows the revaluation of the city's Cultural Heritage through the landscape values present in it.

Keywords: Values, Landscape, City, Cultural heritage.

\section{Introducción}

Al hacer referencia a los valores presentes en el paisaje de la ciudad de Pachuca, se infiere que existen y, por tanto, están ahí; sin embargo su presencia no siempre es detectada por sus habitantes. La actual administración municipal (2017-2020) bajo el slogan "Pachuca, La Casa de Todos" presentó un proyecto denominado Ruta Arqueológica Minera (2017) en el que realizó algunas entrevistas a la población que han sido registradas en documentos y videos presentados por esa dependencia; en ellos se detectó que algunas personas entrevistadas, elegidas al azar, desconocen o tienen poco conocimiento de los elementos que componen el paisaje de su ciudad, por lo que se consideró que su capacidad para identificar, reconocer y valorar su territorio es baja.

Esta supuesta falta de conocimiento acerca de los valores presentes en la ciudad y lo que significan, obliga a mostrar el sentido del concepto de valor y su relación con los elementos que componen el paisaje para que puedan ser descubiertos, conocidos y apreciados por la población que, al ser consciente de ellos, pueda dimensionar y posiblemente comprometerse con su tutela, mantenimiento y conservación.

\section{Elementos metodológicos}

Para el desarrollo de esta etapa de la investigación se ha tomado como referencia el método de análisis de la Metropolitan Situation Analysis (MESA), concebido mediante el programa "Training for Education, Learning and Leadership Towards a New Metropolitan discipline TELLme", que pertenece a la red internacional de investigación sobre ciudades y metrópolis; actualmente es coordinada por la Facultad de Arquitectura del Politécnico de Milán, Italia, bajo el auspicio del Programa Erasmus Plus de la Unión Europea, (2014-2020) en el cual también participa la Universidad Autónoma Metropolitana-Unidad Azcapotzalco, con el programa de posgrado de Diseño, Planificación y Conservación de Paisajes y Jardines. Este procedimiento se realizó con acuerdos entre la Comunidad Europea, mismos que han servido también para la realización de documentos como, entre otros, el Catálogo de Paisajes de la Región Metropolitana de Barcelona (2017).

A partir de lo anterior se detectó la necesidad de describir los atributos, valores y retos que existen en el territorio en estudio y ello precisa de una exhaustiva recopilación de información documental, bibliográfica, cartográfica y fotográfica; además de realización recorridos por la ciudad y de entrevistas a integrantes de la población local con el fin de definir los objetivos de calidad paisajística que puedan servir como punto de encuentro entre las aspiraciones de la ciudadanía.

Las fuentes consultadas fueron el Archivo General del Estado de Hidalgo, la Biblioteca Central de la Universidad Autónoma del Estado de Hidalgo, el Archivo Fotográfico Casasola ubicado en el Exconvento San Francisco, las oficinas del Servicio Geológico Mexicano, el Museo de Minería, que también es Sede Honoraria del Comité Internacional para la Conservación del Patrimonio Industrial (TICCH); todas ellas ubicadas en la ciudad de Pachuca. 
Para la realización de los recorridos se acudió a la Fundación Arturo Herrera Cabañas; el personal capacitado fungió como guía y apoyo para ubicación de puntos estratégicos a visitar en las caminatas por los barrios altos de la ciudad. También con apoyo del personal de la Presidencia Municipal y del Consejo Nacional de Estudiantes de Arquitectura CONEA, se visitó el Barrio Fundacional de Camelia en el que se pudo entrevistar a pobladores con varias generaciones de arraigo en el territorio.

Tras la revisión del material recopilado, se procedió a registrar la información en mapas digitales obtenidos del Instituto Nacional de Estadística y Geografía (INEGI, 2015). Para la realización de la cartografía con georreferencia de los datos resultantes se utilizó el software QGIS versión 3.10, concentrando la información por atributos, clasificándolos de acuerdo con la metodología citada para mostrar los elementos de valor natural, histórico y estético mayormente reconocidos que componen el área que se ha estudiado.

\section{Sobre la noción "valor del paisaje"}

No es la intención de este artículo presentar una exegesis del concepto de "valor presente en el paisaje"; sin embargo es necesario partir de algunas definiciones e ir concatenando las ideas para una mejor comprensión del mismo. Según el Gran Diccionario Enciclopédico Ilustrado (1972), el término proviene del latín (valor, óris) y significa grado de utilidad o aptitud de las cosas; también fuerza o virtud de las cosas para producir sus efectos. Estos términos van permitiendo comprender que aquello a lo que se da o asigna un valor es porque contiene un grado de utilidad o aptitud que produce un efecto. Encaminando el concepto hacia "su puesta en valor o valorización" se observa que González-Varas (2008) lo analiza partiendo de un término francés (mettre en valeur o mise en valeur), también del italiano (valorizzazione) y del inglés (enhagement) "valorizar" o "poner en valor" y directamente lo aplica a un bien cultural determinado para designar el conjunto de acciones inmateriales para aplicar una "tutela activa" sobre ese bien. De lo anterior se puede deducir que, si existe algo que tiene un valor, éste debe ser tutelado o custodiado de alguna forma y podría presumirse el procurar conservarle.

A principios del siglo XX Aloïs Riegl (1903, reeditado 1999) un erudito en términos de filosofía, historia y artes definió con precisión el concepto de valor en un documento que llamó Der Moderne Denkmalkultus (El Culto Moderno a los Monumentos), aplicándolo como "Valores Monumentales" hacia bienes inmuebles o tangibles considerando su evolución histórica, rememoración, antigüedad y contemporaneidad dado que era necesario ya en esa época analizar la condición de valoración, catalogación, cuidado, mantenimiento o grado de deterioro de los bienes que se debían considerar para decidir las acciones a tomar sobre ellos.

Busquets y Cortina (2009) enunciaron en su Manual de protección, gestión y ordenación del paisaje, el concepto del "valor Paisajístico" como una cualidad o conjunto de cualidades que las sociedades atribuyen a los paisajes y los clasifican, entre otros, en productivos, ecológicos sociales, estéticos e históricos, haciendo mención a que esta valoración depende del ideario de cada sociedad, así como del momento histórico específico y que ciertamente con acciones y proyectos de gestión encaminados a su regulación, integración paisajística o restauración pueden mejorar o incrementar su calidad, aumentando así su valor. Estos conceptos dan pautas para analizar las características de los bienes actuales, no sólo los inmuebles tangibles sino también los intangibles, incluyendo sus entornos.

En la última década, organismos especializados como El Consejo Internacional de Monumentos y Sitios (ICOMOS), la Federación Internacional de Arquitectos Paisajistas (IFLA) y la Iniciativa Latinoamericana del Paisaje (LALI) analizan y presentan tanto problemáticas como propuestas y proyectos para la mejora del territorio, el paisaje y el patrimonio. Se percibe la relevancia de estos temas y el trabajo arduo que se realiza para 
comprender mejor las definiciones, atributos, clasificación de valores y procedimientos para la gestión de los mismos en los distintos países. Se observa que el paisaje es percibido de manera integral; sin embargo, para poder comprenderlo como tal es necesario separar y reunificar las partes que lo conforman hasta lograr percibirlo como un todo.

En las páginas que siguen se pretende mostrar algunos de los atributos, utilidades y aptitudes, presentes en la zona en estudio en las que se puede apreciar la existencia de esa fuerza en el territorio que ha producido efectos tangibles para procurar, ante propios y extraños, poner en valor a la ciudad minera de Pachuca, dado que cuenta con los atributos suficientes evidentes en su evolución natural e histórica para ser reconocidos por su valor paisajístico.

\section{Resultados}

\section{Valor natural}

De acuerdo con el Catálogo de Paisaje de Cataluña (2016) este valor muestra los factores o elementos que determinan la calidad del medio natural, los paisajes reconocidos legalmente por criterios netamente naturales y los espacios con un interés especial ya sea éste natural o ecológico, por su representatividad o singularidad. Este valor permite ver en el paisaje información referente al uso que se le ha dado al lugar y cómo ha sido transformado así como los motivos que sus habitantes han tenido para modificarlo y el significado que le han atribuido al mismo.

Según el Instituto para el Federalismo y el Desarrollo Municipal (2010), la Zona Metropolitana de Pachuca (ZM-P) se encuentra sobre la provincia fisiográfica del eje Neovolcánico [20 07’ 18" latitud norte y $98^{\circ} 44^{\prime} 09^{\prime \prime}$ latitud oeste], con una altitud entre 2400 y 3000 msnm, en la Sierra de Pachuca que forma parte de la Sierra Madre Oriental de México. Está compuesta por siete municipios: Pachuca de Soto, Mineral del Monte, Mineral de la Reforma, Epazoyucan, Zempoala, Zapotlán de Juárez y San Agustín Tlaxiaca.

\section{Sitios reconocidos}

Cabe destacar que, desde el 5 de mayo del 2017, la UNESCO reconoció el Geoparque Comarca Minera en Hidalgo con 31 geositios de interés tanto para la investigación como para el desarrollo económico del territorio a través del ecoturismo. Esta declaratoria también incluyó una propuesta para la creación de un comité nacional que regule dichos trabajos; como avance a esta solicitud, en noviembre del 2019 se realizó una reunión encaminada a integrar ese comité en el Consejo de Ciencia, Tecnología e Innovación de Hidalgo (CITNOVA) ubicado en el municipio de San Agustín Tlaxiaca que también forma parte de la Zona Metropolitana de Pachuca (ZM-P).

El Geoparque está conformado por nueve municipios del Estado (Figura 1), de los cuales cuatro forman parte de la ZM-P; y son Mineral del Monte, Pachuca de Soto, Mineral de la Reforma y Epazoyucan, distribuidos en estos se encuentran 15 de los 31 geositios definidos: 8 monumentos antrópicos y 7 de valor natural.

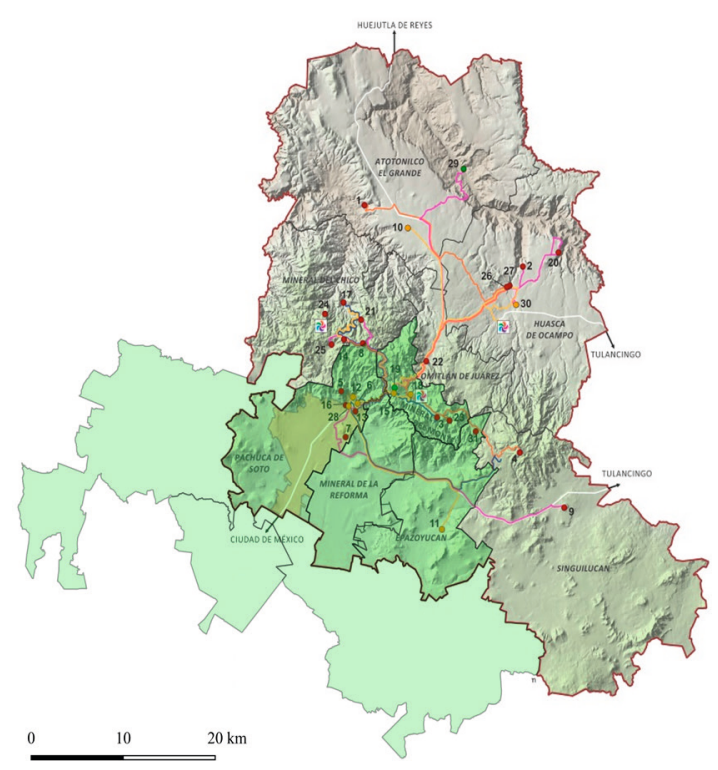

Figura 1. Mapa que muestra la confluencia entre el Geoparque Comarca Minera con reconocimiento UNESCO y Zona Metropolitana de Pachuca. Fuente: elaboración propia. 
Los geositios naturales por municipio son:

\section{Mineral del Monte:}

- Cantera de Tezoantla, material de color blanco con tonos verdosos que se utilizó en la construcción de varios edificios históricos, incluida la Torre del Reloj Monumental de Pachuca.

- Parque Estatal Bosque el Hiloche de composición mixta, predominio de coníferas (protegido desde 2004).

- Peñas Cargadas, rocas en equilibrio inestable (rocas volcánicas riolitas).

\section{Pachuca de Soto Hidalgo:}

- Depósitos Tobáceos de Cubitos, compuestos de lava (basalto, andesíta, tobas y brechas).

- Mirador del Cerro del Lobo, ecosistema urbano actual (lavas y brechas andesíticas) y alteración hidrotermal.

- Cerro de San Cristóbal, centenaria localidad mineralógica (cristobalita y tridimita, rocas volcánicas andesita).

\section{Epazoyucan:}

- Cerro de las Navajas (compartido con el Municipio de Singuilucan) rocas volcánicas: obsidiana, pómez y riolita.

- Traquita el Guajolote, erosión caprichosa de una roca singular, rocas volcánicas traquita.

Además de los lugares descritos, la ZM-P cuenta al norte con El Parque Ecoturístico El Cerezo que está compuesto de grandes valles, zonas arboladas principalmente con oyameles (Abies religiosa) y encinos (Quercus sp), lagunas y grandes extensiones de pasto, conjuntos de rocas con formas caprichosas de singular belleza; en él se encuentran zonas conocidas como el Valle de los Enamorados y el Llano Grande; es considerado también parte del Parque Nacional El Chico (Figura 2), que a partir del 6 de julio de 1982 cuenta con su declaratoria, misma que se publicó en el Diario Oficial de la Federación con su Programa de Manejo (Ante- cedente DOF 13 de septiembre de 1922 decretado como "Reserva Forestal Nacional"). Este parque cuenta con una superficie de 2,739.0263 hectáreas y está conformado por los municipios de Mineral del Chico $^{1}$, Atotonilco el Grande, Mineral del Monte y Pachuca de Soto.

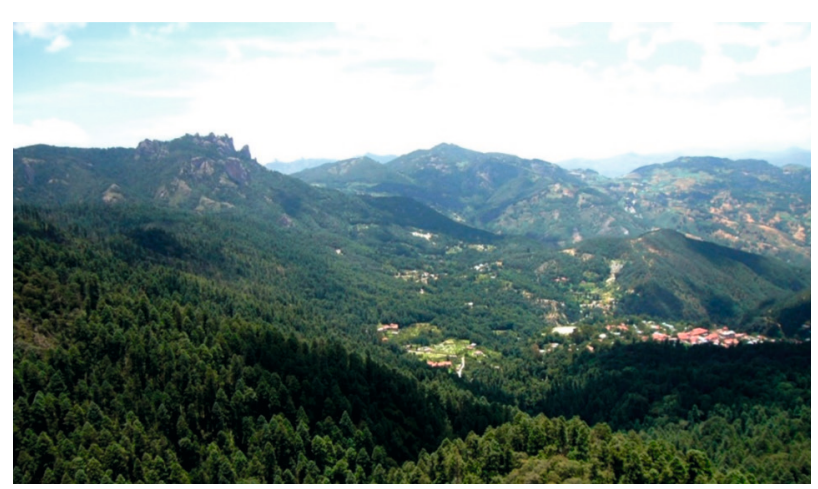

Figura 2. Parque Nacional El Chico, Hidalgo. Fuente: Comisión Nacional de Áreas Naturales Protegidas. Fuente: Gobierno de México (s.f).

\section{Ejidos}

En la ZM-P existen ocho ejidos o comunidades agrarias: Venta Prieta, El Cerezo, El Desmonte, Huixmi, Matilde, Santiago Tlapacoya, Santa Julia y Aquiles Serdán; son los territorios que aún están constituidos legalmente como áreas de cultivo o de actividad rural en los que se produce maíz, fríjol, cebada, trigo, tuna, nopal, alfalfa y maguey. Sin embargo, en el Periódico Oficial del Estado, a partir del 17 de septiembre del año 2007, está publicada la "Ley de asentamientos humanos, desarrollo urbano y ordenamiento territorial del Estado de Hidalgo", que continua vigente ${ }^{2}$ lo cual permite que, mediante el procedimiento legal correspondiente, los terrenos puedan cambiar su uso de suelo y convertirse en configuración urbana

1 Mineral del Chico y Atotonilco el Grande no pertenecen a la zona metropolitana de Pachuca, pero colindan con ella al norte.

2 La última revisión y reformas a la Ley de asentamientos humanos, desarrollo urbano y ordenamiento territorial del Estado de Hidalgo fue publicada en el Periódico Oficial el 14 de mayo del 2018. 
para uso comercial, habitacional e industrial. También pueden ser expropiados por causa de utilidad pública mediante decretos aprobados por la federación (DOF, 2016), como está establecido en la Ley Agraria en los art. 93 y 94 (fracciones II, V y VIII) y art. 5 fracción VI de la Ley General de Asentamientos Humanos, para ser utilizado bajo el rubro de donación para equipamiento, infraestructura y servicios urbanos que el municipio controla.

\section{Parques urbanos}

Estas extensiones de territorio, delimitadas en manzanas dentro de la traza urbana, contribuyen a mejorar la calidad de vida de los habitantes de la ciudad; en ese sentido la Organización Mundial de la Salud (OMS), recomienda que por cada habitante debe haber entre 10 y $15 \mathrm{~m}^{2}$ de área verde y/o ajardinada, dato referido por Escolástico et al (2015). En la zona metropolitana se cuenta con parques, jardines y espacios con áreas verdes, intentando acercarse a este requerimiento. Sin embargo, algunos de ellos destacan del común de áreas verdes dado que existen registros históricos de su presencia en el territorio; tal es el caso del Parque Hidalgo, uno de los espacios naturales emblemáticos de la ciudad en virtud a que la superficie que actualmente ocupa tiene referencia del año 1596 de haber pertenecido a los huertos del exconvento franciscano hasta que, en 1861 de acuerdo con las Leyes de Reforma, se expropian las instalaciones y anexos a edificios religiosos. El 4 de octubre del año siguiente se firma un contrato entre un particular, José Luis Revilla, y el Síndico del Ayuntamiento de Pachuca, José María de los Cobos, para realizar un jardín que sirviera de paseo a la manera de la Alameda Central en la Ciudad de México; el proyecto se concluyó veinte años después por el ayuntamiento de Pachuca con el nombre del entonces presidente Porfirio Díaz; se llama Parque Hidalgo desde el 14 de abril de 1911 (Pachuca brilla, 2017). En la Figura 3 se muestra el pórtico principal de acceso al parque, que aún hoy puede ser visitado.

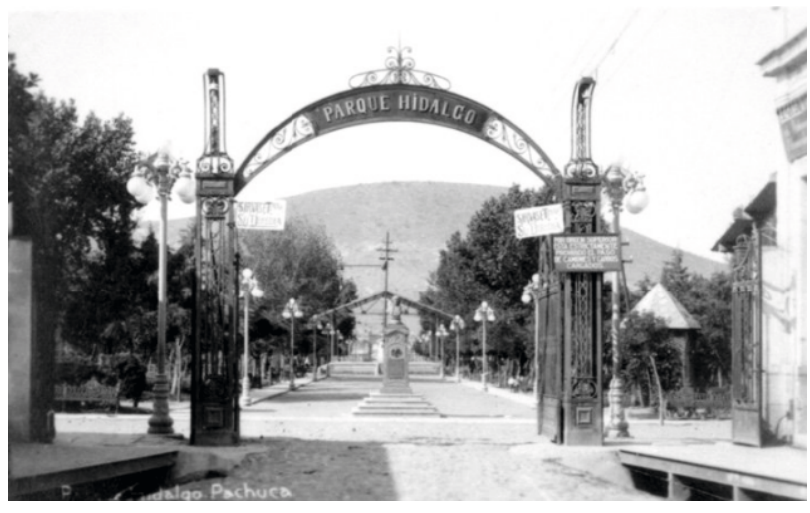

Figura 3. Puerta Principal del Parque Hidalgo 1925. Fuente: Mediateca INAH.

En la misma zona destacan dos jardines que se pueden relacionar con el Parque Hidalgo puesto que también se ubican en los alrededores del exconvento de San Francisco que durante el siglo XIX fue el Hospital de Mineros. A manera de plaza de acceso se encontraba un jardín que contaba con una fuente y distribución radial con arcos de madera en los accesos. A partir de 1956 se renombra como Jardín Bartolomé de Medina. Se encuentra enfrente de la Fototeca Nacional conocida por albergar el acervo "Casasola" del fotógrafo con el mismo apellido; actualmente se han sumado colecciones de diversos fotógrafos que con sus imágenes documentaron la historia de México a finales del siglo XIX y principios del XX. En la actualiad en este hermoso jardín se encuentra la entrada de la escuela de música de Bellas Artes. El jardín Luis Pasteur se ha fragmentado del Parque Hidalgo, pero antiguamente formaba parte de la misma extensión de los huertos citados; se puede visitar frente a las instalaciones del centro de Salud de la Secretaría de Salubridad y Asistencia Pública sobre la avenida Madero y frente al antiguo Hospital Civil y Escuela de Medicina de la Universidad Autónoma del Estado de Hidalgo (UAEH) por la calle Dr. Eliseo Ramírez Ulloa. Cuenta con áreas ajardinadas, juegos infantiles y fuente central.

Otros jardines a destacar se muestran en la Figura 4 y se encuentran ubicados sobre la calle Mariano Abasolo 600; han formado parte del paisaje desde el siglo XVIII cuando se construyó una iglesia a la 
virgen de Guadalupe en 1725. Durante ese tiempo fueron ocupados como panteón pues en esa época se consideraba cercano al templo llamándole "camposanto". Actualmente el edificio ha sido rehabilitado para ser la Sala de Actos Solemnes "Baltazar Muñoz Lumbier" del Centro Cultural La Garza de la UAEH; el cuerpo horizontal del inmueble que lo conforma ha tenido diferentes usos a lo largo de su historia, siendo primero el Hospital de San Juan de Dios que atendió enfermos, mineros y personas con bajos recursos; posteriormente se convirtió en el Instituto de Artes y Oficios y hacia el año 1869 en el mes de marzo, se consolidó su vocación académica como el Instituto Científico y Literario del naciente Estado de Hidalgo, quedando sus jardines abiertos para recibir al público en general. Se ha vuelto una tradición universitaria iluminarlos cada año en los primeros días del mes de diciembre, hecho muy esperado por la población dada la convivencia popular que se desarrolla alrededor de ellos (UAEH, 2014).

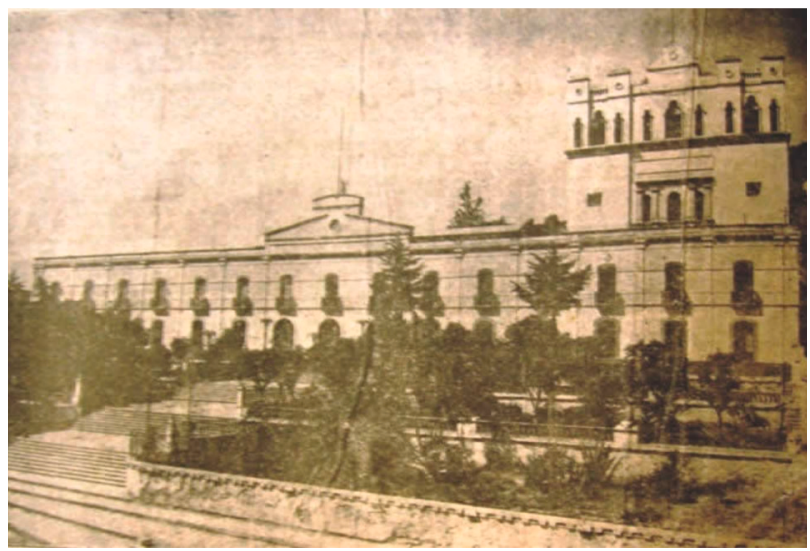

Figura 4. El Instituto Científico y Literario de Pachuca, Hidalgo. Fuente: El Observador (1931, p. 2).

Es importante destacar que aún cuando se describen atributos con valor natural, éstos también tienen un valor histórico pues son hechos registrados en crónicas, periódicos y fotografías, y tienen además un valor simbólico pues son espacios que algunos lugareños reconocemos por haber vivido ahí alguna experiencia personal. Esta es una de las observaciones que los expertos hacen al reconocer que un paisaje no se percibe desmembrado, sino en forma integral, con todos sus atributos.

Teniendo en cuenta la metodología utilizada, la información recabada ha sido georreferenciada en un mapa obtenido en INEGI, referente a la entidad federativa de Hidalgo, codificada con el número 13 específicamente la zona metropolitana de Pachuca; la clasificación muestra los atributos presentes en el valor natural, el avance hasta la fecha se muestra en el Mapa 1. Es importante decir que esta información genera por cada capa una tabla con datos que permite observar identificación, nombre, descripción, datos estadísticos, alturas, áreas, fechas y fuentes de obtención de datos para consultas específicas.

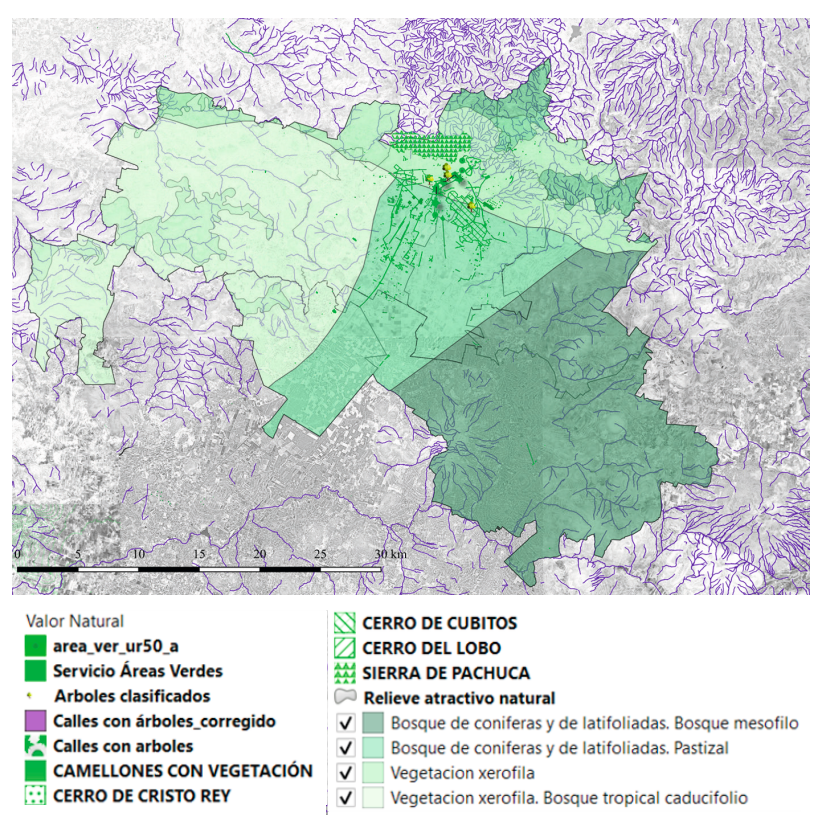

Mapa 1. Valor Natural en la zona de estudio. Fuente: elaboración propia con base en QGIS 3.10.

\section{Valor histórico}

Según el Catálogo de Paisaje de Cataluña (2016) este valor se percibe en la huella que va dejando el ser humano a través de los hechos que registra la historia en el lugar, en el uso y transformación que 
le ha dado al espacio natural. La minería ha sido la vocación inicial de la región y para explotarla ha sido necesario introducirse en el territorio, excavar, penetrar para formar túneles, utilizar madera de los árboles de la región para hacer estructuras de apoyo interno y externo, sangrar al río para redirigir su trayectoria a las haciendas de beneficio donde se procesa el mineral; todo esto ha hecho cicatrices en la tierra que hoy son posibles de apreciar así como en su traza urbana y edificaciones que dan cuenta de cada etapa histórica, fiel testimonio del diario vivir de la ciudad de Pachuca.

Según Ruiz et al (2012) hacia el año 1500 la región estuvo habitada por grupos indígenas como otomí y náhuatl; en ese tiempo la principal actividad mineral estaba enfocada en la extracción de la obsidiana en el denominado Cerro Navajas. Ese material era muy valorado porque servía como herramienta de corte y para elaborar puntas de flechas, armas y utensilios diversos.

Según la reseña escrita por la Junta Corresponsal del Estado de Hidalgo (1884), en el año 1555 el minero español Bartolomé de Medina en la Hacienda de Beneficio la Purísima Concepción, fue descubridor del beneficio de los metales por un procedimiento llamado de amalgamación o beneficio de patio; este descubrimiento hizo más accesible el trabajar los metales, hecho que fuera conocido en América y Europa dada la gran cantidad de mineral beneficiado que se reportó a la Corona.

De acuerdo con Ruíz et al (2012) la pintura de Cempoala fechada en 1580 (Figura 5) marca la presencia de un señor español y un indígena que indican la supervisión del trabajo minero. Cabe señalar que este códice también es testigo de la presencia de árboles de encino que son representados a lo largo del margen lateral izquierdo. Se dice que el señorío de Cempoala fue uno de los aliados de Hernán Cortés, a quien los naturales trataron con hospitalidad dado que rendían tributo al pueblo azteca y vieron en el conquistador un aliado para su "libertad" (Lorenzo-Monterrubio, 2012). La Junta Corresponsal del Estado de Hidalgo (1884) también reporta que hacia el año 1682 en la Crónica de la Provincia de San Diego impresa en México, se conoce que el nombre de la ciudad proviene de Pachoa, término mexicano que significa apretura o estrechez, dada la configuración de las dos montañas donde se asienta la población que forman una cañada estrecha, que fue llamada portezuelo.

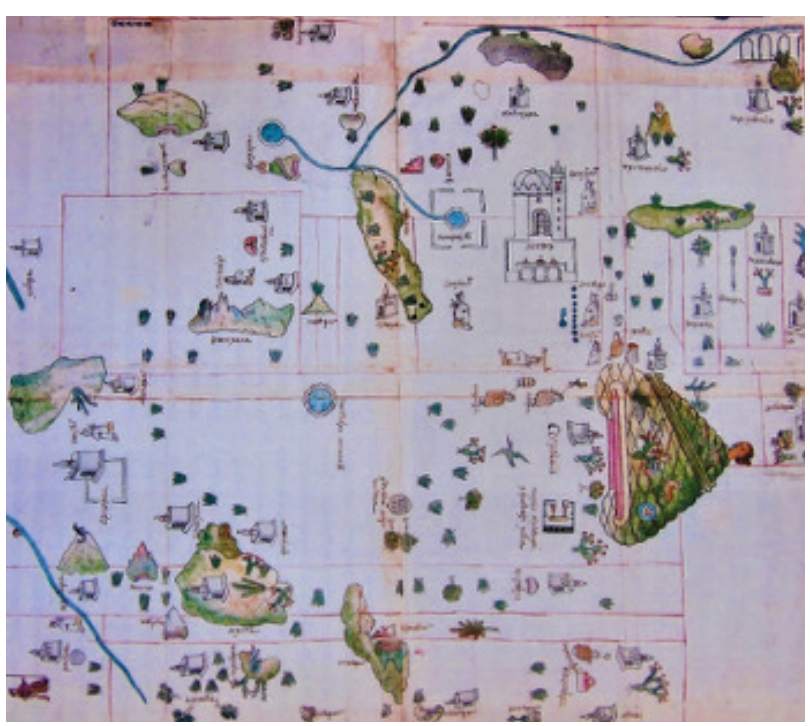

Figura 5. Pintura de Cempoala 1580 que muestra la zona de Pachuca en la esquina inferior izquierda. Fuente: Ruiz et al (2012).

A mediados del siglo XVIII se representa la región entre montañas verdes, como se observa en la Figura 6, con construcciones españolas y haciendas de beneficio donde se procesaba el mineral extraído; también se construyeron lugares para almacenar el mineral antes de su embarque denominados las Cajas Reales para la administración de la extracción minera, así como las casonas de los administradores y las iglesias católicas de La Asunción de la Virgen en la Plaza Real y en el límite sur, el conjunto de iglesia y convento de la Orden Franciscana. 


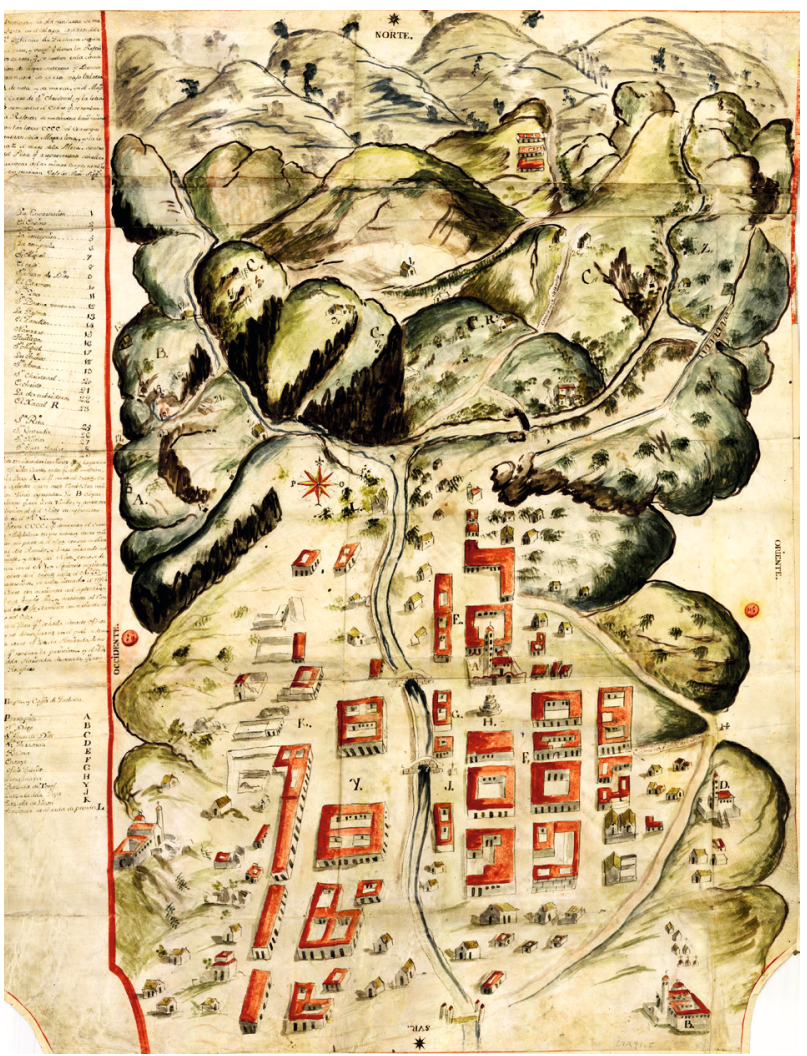

Figura 6. Mapa de los Cerros del Real de Minas de Pachuca, 1750. Fuente: Mapoteca Orozco y Berra.

De acuerdo con Viornery (2006) para el año 1823 Manuel Romero de Terreros, Tercer Conde de Regla, anuncia en renta las minas puesto que se encuentran inundadas y él no cuenta con tecnología para trabajarlas, por esta razón llegan mineros ingleses y la forma de trabajo cambia al utilizar maquinaria de vapor.

La percepción del territorio en las montañas se ve modificada ya que son construidos los edificios para la producción minera y el sangrado del río para utilizar el agua en el proceso minero. A juzgar por los documentos fechados en año de 1864 es un periodo histórico importante (Ruíz et al, 2012) dado que se realizan varios planos de diferentes tipos para referenciar el número de minas y su posición detallada; también para mostrar la traza urbana a la fecha y en otros más, se marcan los tramos de túneles que son conocidos y ubicados como vetas a ser explotadas.

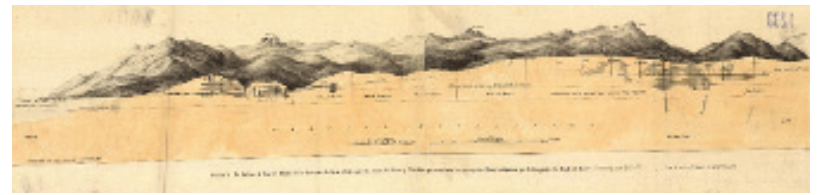

Figura 7. Perfil No. 1 de Pachuca a Real del Monte en la dirección de Oeste a Este. Fuente: Compañía Real del Monte y Pachuca.

Menes-Llaguno (2006) menciona que tras la Independencia del país este territorio fue denominado Estado de México; sin embargo, indica que hacia el año 1865 se dispuso la división territorial que determinó la erección del Estado de Hidalgo, hecho que se concretó el 16 de enero de 1869, siendo el primer gobernador el Lic. Juan Crisóstomo Doria y quedando la ciudad de Pachuca como la capital del Estado. En 1884 la Junta Corresponsal del Estado (JCEH) elaboró una reseña relativa al entonces reciente Estado de Hidalgo que se presentó en la Exposición Universal de Nueva Orleans; en este documento se explica que se toma la decisión de subdividir el territorio para poder "administrar de mejor manera las riquezas en él, existentes”. Dicho documento fue realizado en la imprenta del Gobierno del Estado ya ubicada en Pachuca, en esta reseña se describieron las principales características del territorio enlistando las vetas encontradas en cada mina, así como su ubicación y producción de minerales; el libro que contiene estos registros puede consultarse en el Archivo General de la Universidad Autónoma del Estado de Hidalgo.

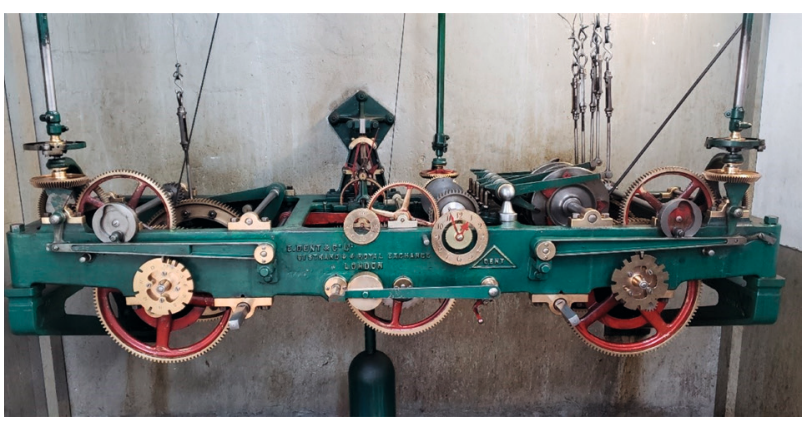

Figura 8. Maquinaria en el interior de la Torre del Reloj Monumental de Pachuca. Fuente: elaboración propia. 
Uno de los símbolos que sí son conocidos de la ciudad es sin duda la Torre del Reloj Monumental, situada en la antigua Plaza de las Diligencias, hoy Plaza Independencia. Lo que tal vez no se sabe es su valor intangible relacionado con las emociones que se producen en el sentimiento y pensamiento de la población en torno a acontecimientos específicos; es posible imaginar el orgullo de los habitantes que desde 1904 vieron la transformación de este lugar y haber estado presentes esa noche del 15 de septiembre de 1910 en la inauguración de la Torre con un gran reloj cuya maquinaria fue fabricada por la empresa DENT (s.f.) que también realizara la del famoso Big Ben de Londres. Cabe destacar que esta maquinaria es única en América, símbolo del poderío económico de la región denominada la Comarca Minera y con este hito tuvo la encomienda de conmemorar el Centenario de la Independencia de México, a petición del entonces presidente de la nación Porfirio Díaz que solicitó la creación de monumentos en las principales ciudades del país. Desde entonces ha sido el lugar de referencia de propios y visitantes, para preservar evidencias de hechos relevantes registrados en fotografías históricas incontables.

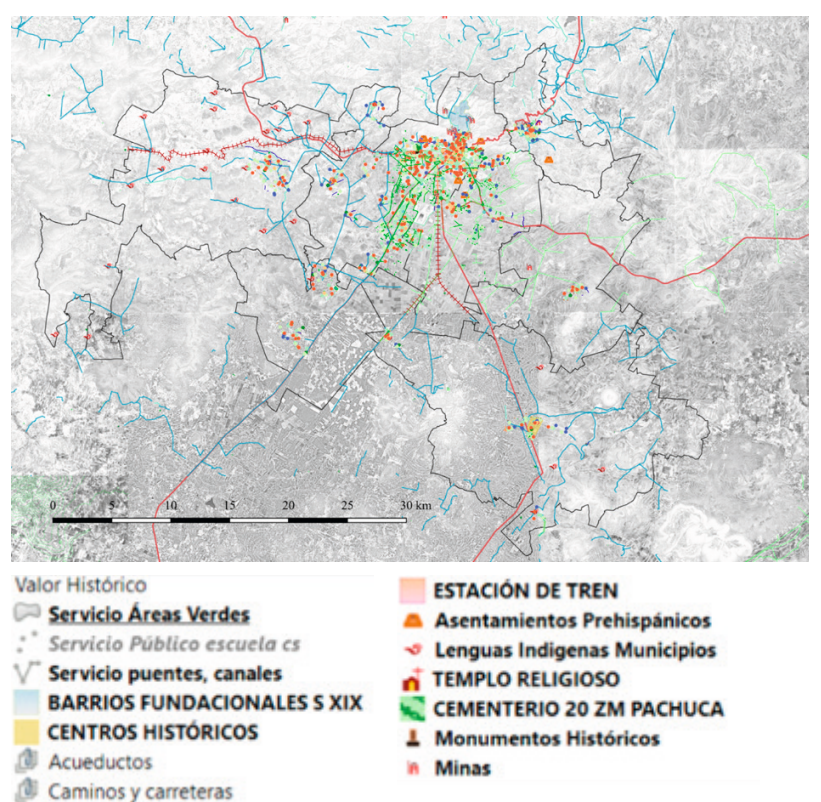

Mapa 2. Valor histórico en el área de estudio (QGIS 3.10). Fuente: elaboración propia.
Se han mostrado algunos de los valores presentes en la ciudad de Pachuca con la intención de que, así como se dicta en la Carta Mexicana del Paisaje $\mathrm{e}^{3}$, sea factible describir su paisaje como esa extensión de territorio percibida por las personas que la observamos y la habitamos y reconocer, asimismo, que la acción e interacción de factores humanos impactan en la naturaleza que nos rodea y determina el carácter de nuestra ciudad.

\section{Conclusiones}

En este artículo se ha procurado presentar una alternativa para refrescar el conocimiento de los habitantes de Pachuca sobre sus valores paisajísticos. Y para lograrlo se han descrito algunos de sus sitios paradigmáticos para reconocer, a través de datos registrados, los hechos históricos que dejaron un testimonio de su relevancia como la Torre del Reloj Monumental, ubicada en Plaza Independencia. Todavía esos lugares pueden ser visitados para buscar esa conexión con sus etapas históricas que hablan de quienes hemos sido, para comprender el desarrollo de esta ciudad y de su primigenia actividad que sigue siendo, aunque en menor escala, la minería.

De esa manera se han compartido como testimonio algunos de los dibujos, planos, perfiles y mapas que conforman la cartografía de base y que muestran una parte de la transformación del territorio para conformar el paisaje minero, que se entretejió entre la naturaleza de la Sierra de Pachuca, las vetas de los minerales y la traza urbana resultante; esta información se ha georreferenciado para poder ser utilizada en futuras gestiones que contribuyan a la conservación de su patrimonio.

Es importante también divulgar los logros conseguidos en cuanto a la valoración internacional ya obtenida como es el Geoparque Comarca Minera, en el que está latente la posibilidad de conocer

3 Signada por la Sociedad de Arquitectos Paisajistas de México, A. C, reconocida también por la UNESCO y el Convenio Europeo del Paisaje. 
y participar en el proceso para la creación del comité nacional recomendado por la UNESCO. Sin embargo, aún es necesario difundir este reconocimiento para que no quede restringido sólo a los especialistas sino que llegue a la población en general pues así podrá conocer esos atributos, apropiarse de ellos y legitimarlos como valores.

Lo anterior se logrará fortaleciendo la educación patrimonial que deberá ser incluida en los planes de gestión pública de la Comarca Minera para permitir la valoración de los elementos presentes en esta ciudad; así la población tendrá más elementos para percibir el paisaje en forma integral, identificarse y comprometerse con él para, posiblemente, formar parte de colectivos y realizar las acciones pertinentes encaminadas a permitir la conservación de sus valores aún presentes para las nuevas generaciones.

\section{Referencias bibliográficas}

Ayuntamiento de Pachuca (7 de noviembre 2017) Ruta arqueológica minera de Pachuca. Recuperado de [https://www.youtube.com/ watch?v=IADnQbkHKXU].

Busquets, J. y Cortina, A. (coordinadores) (2009). Gestión del paisaje. Manual de protección, gestión y ordenación del paisaje. Madrid: Ariel.

Cárdenas, E. (1972). Gran Diccionario Enciclopédico Ilustrado. New York: Selecciones del Reader's Digest.

DENT (s.f.) A Legacy Of Horological Excellence Watch, Chronometer and Clock Maker. Since 1814. Dent, London. Recuperado de: [http:// www.dentlondon.com/about/history.php]. [acceso: 10/07/2019].

DOF (2006). Decreto por el que se expropia por causa de utilidad pública una superficie del ejido Santa Matilde, Municipio de Pachuca de Soto, Hgo. Diario Oficial de la Federación, 28 de noviembre de 2006. Recuperado de: [http:// www.dof.gob.mx/nota_detalle.php? codigo $=49$ $38599 \&$ fecha $=28 / 11 / 2006$ ].
El Observador (1 de julio de 1931). Instituto Científico y Literario de Pachuca, Hidalgo. Periódico El Observador, p. 2.

Gobierno de México (s.f.). Comisión Nacional de Áreas Naturales Protegidas. Recuperado de: [https://www.gob.mx/conanp/articulos/ parque-nacional-el-chico-belleza-ecologica114969?idiom=es... [acceso: 02/12/2018].

Diario Oficial de la Federación DOF (6 de julio de 1982), Decreto "Parque Nacional El Chico". Recuperado de: [http://dof.gob.mx/nota_ detalle_popup.php?codigo $=5107107]$ [acceso: 02/12/2018].

Diario Oficial de la Federación DOF (13 de septiembre de 1922), Decreto "Reserva Forestal Nacional". Recuperado de: [http://www.dof. gob.mx/nota_detalle.php? codigo $=4797392 \& \mathrm{f}$ echa $=24 / 06 / 1986$ ] [acceso: 02/12/2018].

Escolástico León, C., Lerma Ruíz, J. A., López Llorens, J. y Alia Sánchez, M. L. (2015). Medio Ambiente y Espacios Verdes. Madrid: UNED.

Generalitat de Catalunya (2017) Catálogo de Paisaje de la Región Metropolitana de Barcelona. Barcelona: Departamento de Territorio y Sostenibilidad y Observatorio del Paisaje de Catalunya.

González-Varas, I. (2008) Conservación de Bienes Culturales. Teoría, historia, principios y normas. Madrid: Cátedra.

Instituto Nacional de Estadística y Geografía (2015) Territorio del Estado Hidalgo según Conteo de 2015. Recuperado de: [http://cuentame.inegi. org.mx/monografias/informacion/hgo/territorio/div_municipal.aspx?tema $=$ me $\& e=13]$ [acceso: 15/10/2018].

Instituto para el Federalismo y el Desarrollo Municipal (2010). Enciclopedia de municipios y delegaciones de México Estado de Hidalgo, Secretaría de Gobernación. Recuperado de: [http://siglo.inafed.gob.mx/ enciclopedia/EMM13hidalgo/index.html]. [acceso: 12/09/2018]. 
Junta Corresponsal del Estado de Hidalgo (1884). Reseña Relativa al Estado de Hidalgo para la Exposición Universal de Nueva Orleans 18841885. Pachuca: Imprenta de Gobierno del Estado.

Lorenzo-Monterrubio, A. y Lorenzo-Monterrubio, C. (2012) Zempoala. Veinte días. Pachuca de Soto: Gobierno del Estado de Hidalgo.

Menes Ll, J. M. (2006) Historia mínima del estado de Hidalgo. México: M.A. Porrúa.

Pachuca Brilla (2017). Parque Hidalgo, con mucha historia. Recuperado de: [https://pachucabrilla. com/parque-hidalgo-con-mucha-historia/].

Periódico Oficial del Estado de Hidalgo (2007) Ley de Asentamientos Humanos, Desarrollo Urbano y Ordenamiento Territorial del Estado de Hidalgo.

Riegl, A. (1999) El culto moderno a los monumentos, caracteres y origen. Madrid: Visor.

Ruíz de la Barrera, R., Yta, M., Díaz Orozco, A., Lorenzo-Monterrubio, A., Vergara-Vergara, J. y Menes-Llaguno, J.M. (2012) Pachuca, Plata, Viento y Voluntad, Vetas de su Historia. Tomo 1. México: Grupo Milenio.
Sala i Martí, P. y Grau i Oliveras, J. (2016) Catàleg de paisatge. Regió Metropolitana de Barcelona. Barcelona: Generalitat de Cataluña. Departamento de Territorio y Sostenibilidad.

Universidad Autónoma del Estado de Hidalgo (2014). Las voces de los muros. México: Centro Cultural Universitario la Garza.

UNESCO (2017). México visita el Geoparque Comarca Minera Hidalgo, para analizar proyectos de conservación y aprovechamiento. Servicio de prensa, UNESCO. Recuperado de: [http://www.unesco.org/new/es/media-ser vices/single-view-tv-release/news/unesco _mexico_visita_el_geoparque_comarca_mine ra_hidalgo_p/].

UNESCO (2019). México busca establecer un Comité Nacional de Geoparques e incrementar su registro en la Red Global UNESCO. Recuperado de: [http://www.onu.org.mx/me xico-busca-establecer-un-comite-nacional-degeoparques-e-incrementar-su-registro-en-lared-global-de-la-unesco/].

Viornery, M. (2006) Pachuca-Real del Monte, Esperanza de Inmigrantes. Pachuca: Lito Impresos Bernal. 